\title{
Impact of Health Care Reform on Treatment Options for Degenerative Spinal Disorders \\ George R Cybulski*
}

Chairman, Division of Neurosurgery, John H. Stroger Hospital of Cook County, USA

Associate Professor of Neurosurgery, Northwestern University Feinberg School of Medicine, USA

The Patient Protection and Accountable Care Act (PPACA) of 2010 will have significant impact upon access and quality of health care for patients and reimbursement for providers in the United States including treatment options including those for degenerative spinal disorders.

While demographic projections of an exploding aging population in the United States in the coming decades would indicate concomitant increased occurrence and resultant demand for treatment of degenerative spinal disorders, major components of the PPACA will create downward pressure on utilization of such treatment. Access to providers, anticipated effect of quality metrics on choice of treatment options, and restraints on provider reimbursement will erect significant barriers to patients seeking treatment for degenerative spinal disorders. In addition, ongoing struggles with financing of the United States budget deficit triggering the "sequester" have already resulted in an automatic $2 \%$ reduction in provider fees for Medicare on April $1^{\text {st }}$.

The PPACA will increase access to health care services via expansion of eligibility for Medicaid and establishment of insurance exchanges to cover employees of small businesses. Cost control measures of the PPACA emphasize utilization of primary care providers who will see an increased reimbursement for their services over specialty care and decreased Medicare reimbursement in particular for specialists will no doubt, result in fewer interventions for degenerative spinal disorders. Improved quality of health care services by reducing fragmentation of care through clinical integration is the objective of forming accountable care organizations (ACOs) which are supported by the PPACA in regard to Medicare patients and has been also applied in some regions in the broader sense to population health management.

In 2013, a number of additional measures of the PPACA will join the formation of Accountable Care Organizations that occurred in 2011-2012. Accountable Care Organizations (ACOs) will take the lead as the major vehicle of the PPACA for "bundling" or integrating delivery in a way to address the present fragmented delivery of health care. However, merging of providers (hospitals and physicians) into entities that will share risks and reimbursement will occur outside of ACO formations. At the heart is the "triple aim" of the PPACA of improving quality of patient care while also addressing overall population health (prevention and wellness) all while reducing cost. Antitrust concerns affecting competition with providers outside hospital-provider mergers must be considered as these mergers will increase the monopoly power of regional health care organizations.

One of the key catch phrases of the PPACA is "value over volume". The intent of the PPACA is to channel more care toward lower cost PCPs and away from higher cost specialists. This naivve premise will only establish significant bottlenecks for patients with degenerative spinal conditions at the primary care level wasting time and resources that would be more effectively utilized by specialists in degenerative spinal disorders with access to definitive management tools.

At the same time expansion of eligibility of traditional fee for service programs such as Medicaid and establishing Insurance Exchanges for subsidizing commercial insurance in 2013 will likely continue to propagate traditional volume over value in direct opposition to the aim of the PPACA. This result is based on the economic principle of moral hazard, an insurance phenomenon in which a person shielded from risk (i.e. paying for a significant share of health care) is likely to utilize more of the subsidized service. In addition, in the face of the anticipated addition of 30 million patients with coverage for health care the demand for services will swamp out the already inadequate PCP infrastructure and inhibit the timely movement of patients to specialty care.

Components of the PPACA directed at the goal of achieving value over volume of care will come through use of metrics for delineating value provided through collection of outcomes via the Patient Centered Outcome Institute, reimbursement through bundling, and ValueBased Purchasing. Value-based purchasing will attempt to incentivize health care organizations to reduce readmissions of Medicare patients for example. Likewise, in 2013 the Medicare Bundled Payment Pilot Program for reimbursing providers is scheduled to go into effect. How these proposed entities will affect health care delivery remains to be seen.

These initiatives have already become a boon to health care consulting firms that offer programs for improving efficiency of health care delivery.

Additional potential for effects on utilization of health care services may come through the Independent Physician Advisory Board (IPAB) (proposed for enactment in 2014) which would utilize evidence-based medicine and cost benefit analysis studies to make recommendations for treatment. Information regarding composition of the IPAB has been vague and likely will be a source of continued contention in Congress as previously this concept has been referred to as a "death panel" by some Congressmen. Regardless, this concept is basically a knock-off of the National Institute of Comparative Effectiveness (NICE) of the National Health Service of Great Britain whose net effect has been to ration health care.

The PPACA notwithstanding, degenerative spinal disorders will continue to present a significant challenge for treatment for a number of additional reasons.

Degenerative disease of the spine runs the gamut from degenerative disc and facet disease with attendant symptoms such as pain and stiffness to cervical spondylotic myelopathy and lumbar spinal stenosis with or without scoliosis. As such treatment may require only non-

${ }^{*}$ Corresponding author: George R Cybulski, M.D, MBA, Associate Professor of Neurosurgery, Northwestern University Feinberg School of Medicine, USA, E-mail: grcneurosurg@gmail.com

Received April 08, 2013; Accepted April 10, 2013; Published April 12, 2013

Citation: Cybulski GR (2013) Impact of Health Care Reform on Treatment Options for Degenerative Spinal Disorders. J Spine S2: e001. doi:10.4172/2165-7939.S2 e001

Copyright: (c) 2013 Cybulski GR. This is an open-access article distributed under the terms of the Creative Commons Attribution License, which permits unrestricted use, distribution, and reproduction in any medium, provided the original author and source are credited. 
Citation: Cybulski GR (2013) Impact of Health Care Reform on Treatment Options for Degenerative Spinal Disorders. J Spine S2: e001. doi:10.4172/2165-7939.S2-e001

Page 2 of 2

steroidal anti-inflammatory medication and physical therapy or more extensive surgical decompression, reconstruction, and instrumentation of the spine.

Accordingly, treatment protocols for degenerative spinal disorders are not standardized and involve treatment by a number of different specialties including primary care, sports medicine, physical medicine and rehabilitation, orthopaedic surgery, neurology, interventional pain specialists, and neurosurgery, as well as additional practitioners who provide treatment via manipulation and physical modalities such as chiropractors, physical therapists, etc.

Under the PPACA, PCPs will likely be expected to have a major role in directing patients with degenerative spinal disorders into care pathways. They will require assistance in making these recommendations, and the challenge for specialists who treat degenerative spinal disorders will be in differentiation of the value of their services from those of PCPs. Specialists can invest in tools for patients and PCPs to utilize in following care pathways and directing them to specialty care networks that are centers of excellence in managing degenerative spinal disorders.

Therefore, specialists treating degenerative spinal disorders will need to not only diligently collect their outcome metrics but also demonstrate their value in terms of returning patients to work and improved function in a timely fashion, etc. This significant challenge also presents a significant opportunity for guiding patients and PCPs to the aforementioned specialty care network. Specialists in the treatment of degenerative spinal disorders must demonstrate the efficacy of their offerings over prolonged management and therefore be robust contributors to the measurement of value.

\section{Potential Effects of PPACA on Treatment of Degenerative Spinal Disorders}

1. Decreasing reimbursement available for intervention as primary care is emphasized and reimbursed over specialty care.
2. Disincentive for device (instrumentation) makers as the device tax enacted in 2013 will affect the bottom line of device makers overall including their budget for research and development of innovation in surgical treatment of degenerative spinal disorders.

3. A smaller pool of reimbursement in the Medicare group - largest consumers of care for degenerative spinal disorders will increase competition for both providers and patients to provide and seek care.

4. The IPAB (if enacted) would be expected to further impact the scope and reimbursement of treatment for degenerative spinal disorders by pronouncements on the efficacy of treatment measures.

5. Value always resides in the eye of the beholder with different perspectives and objectives between the consumer and the provider.

\section{Conclusion}

The emphasis of the PPACA of value over volume will present significant challenges for providers of treatment of degenerative disorders of the spine accustomed to volume over value and to utilization of fusion with instrumentation accompanying decompressive laminectomies of the cervical and lumbar spines. Going forward value will have to be demonstrated by outcomes and additional expensive spine instrumentation will have to be justified accordingly. Because of this, the ongoing financial impact of providing increased numbers of citizens with health care under the PPACA will produce additional downward pressure on providing such care producing de facto rationing whether outright by bodies such as IPAB or by following basic economic principles of supply and demand. Improved quality of life for patients with degenerative spinal disorders will lie with development of innovative treatment and delivery methods with value measured by improved mobility and other functional measures.

\footnotetext{
This article was originally published in a special issue, Degenerative Spinal Disorders handled by Editors. Dr. George R Cybulski, Northwestern University Feinberg School of Medicine, USA; Dr. Ely Steinberg, Sourasky TelAviv Medical Center, Israel
} 